\title{
DINÂMICA POPULACIONAL DA MOSCA-NEGRA-DOS-CITROS Aleurocanthus woglumi ASHBY (HEMIPTERA: ALEYRODIDAE) EM Citrus spp. NO MUNICÍPIO DE SÃO LUÍS - MA ${ }^{1}$

\author{
FABÍOLA RODRIGUES MEDEIROS², RAIMUNDA NONATA SANTOS DE LEMOS ${ }^{3}$, \\ ANGELO LUIZ TADEU OTTATI ${ }^{4}$, JOSÉ RIBAMAR GUSMÃO ARAÚJO ${ }^{5}$,
} KENESON KLAY GONÇALVES MACHADO ${ }^{6}$, ANTÔNIA ALICE COSTA RODRIGUES ${ }^{7}$
}

RESUMO-O objetivo deste trabalho foi avaliar a dinâmica populacional de Aleurocanthus woglumi ao longo de um ano, verificando-se a época de maior ocorrência, seus picos populacionais e a distribuição dessa praga em dois pomares de Citrus spp. no município de São Luís - MA. Para o estudo da dinâmica populacional, foram selecionadas 10 plantas ao acaso, em cada pomar, coletando-se 20 folhas por planta, no período de julho de 2006 a junho de 2007, contando-se o número de posturas, ovos e ninfas. Para a determinação da distribuição de $A$. woglumi, as plantas foram divididas em quadrantes: norte, leste, sul e oeste, retirando-se 5 folhas/quadrante, totalizando-se 200 folhas por área de coleta. Com relação à distribuição na planta, o delineamento foi o inteiramente casualizado, adotando-se o esquema fatorial 2 x 4 (período seco e chuvoso x quadrante), com 13 repetições ( $n^{\circ}$ de coletas). Realizou-se uma análise exploratória de dados, e as médias obtidas foram submetidas ao teste de Tukey, a 5\% de probabilidade. Observou-se, nas duas localidades, que o inseto se distribuiu na copa da árvore de maneira homogênea e que os maiores níveis populacionais de A. woglumi ocorreram no período de baixa precipitação (julho a dezembro de 2006).

Termos para indexação: Aleirodídeos, flutuação populacional, citricultura.

\section{POPULATIONAL DYNAMICS OF CITRUS BLACKFLY Aleurocanthus woglumi ASHBY (HEMIPTERA: ALEYRODIDAE) IN Citrus spp. IN SÃO LUÍS, MARANHÃO, BRAZIL}

\begin{abstract}
This paper aims to evaluate the populational dynamics of Aleurocanthus woglumi throughout one year, verifying the season with higher density, its populational peaks and citrus blackfly distribution in Citrus spp. in two orchards, in São Luís, Maranhão, Brazil. To study the populational dynamics, 10 plants were selected at random, in each orchard, as well as 20 leaves per plant, from July 2006 to June 2007, and the number of layings, eggs and nymphs was counted. To determine the vertical distribution of A. woglumi, plants were divided into quadrants: North, East, South and West, and 5 leaves/quadrant were removed, totaling 200 leaves per collecting area. Regarding to distribution over the plant, the delineation was entirely randomized adopting a 2 x 4 factorial scheme (dry and rainy seasons x quadrants), with 13 repetitions (number of collections). It accomplished an exploratory analysis of data and averages submitted to Tukey test at a 5\% probability rate. It was observed that on the two locations the insect was distributed homogeneous on the tree canopies and that the highest populational levels of $A$. woglumi occurred in the low precipitation season (from July to December 2006).
\end{abstract}

Index terms: Aleyrodidae, population fluctuation, citriculture.

${ }^{1}$ (Trabalho 264-08). Recebido em: 21-10-2008. Aceito para publicação em: 28-07-2009. Parte da dissertação do primeiro autor apresentada ao Curso de Mestrado em Agroecologia/UEMA.

${ }^{2}$ Engenheira Agrônoma, Msc. em Agroecologia pela UEMA, São Luís-MA. fabiolaagro@hotmail.com

${ }^{3}$ Prof. Dr. do Depto. Fitotecnia e Fitossanidade/CCA/UEMA - C. P. 9, CEP 65.055-098, São Luís-MA. rlemos@cca.uema.br

${ }^{4}$ Serviço de Sanidade Agropecuária, Superintendência Federal de Agricultura/MA. 65025-500, São Luís-MA, Brasil. angelo.ottati@agricultura.gov.br

${ }^{5}$ Prof. Dr. do Depto. Fitotecnia e Fitossanidade/CCA/UEMA - C. P. 9, CEP 65.055-098, São Luís-MA. gusmão@elo.com.br ${ }^{6}$ Aluno do curso de Agronomia/UEMA- Caixa Postal 9, CEP 65.055-098, São Luís-MA. kenesonk@yahoo.com.br

${ }^{7}$ Prof. Dr. do Depto. Fitotecnia e Fitossanidade/CCA/UEMA - C. P. 9, CEP 65.055-098, São Luís-MA. alicecosta@bol.com.br 


\section{INTRODUÇÃO}

A produção de citros in natura para os mercados interno e externo tem-se destacado pela crescente melhoria da qualidade dos frutos, visto que é uma cultura que está sendo ameaçada pelos altos custos dos insumos e pelo aumento da incidência de pragas, que elevam o custo de manutenção do pomar. As pragas são responsáveis pelo alto custo de implantação, interdição e erradicação de plantas dos pomares citrícolas.

Atualmente, a mosca-negra-dos-citros (Aleurocanthus woglumi Ashby, 1915) vem destacando-se na fruticultura brasileira, afetando um grande número de plantas como citros, cajueiro, abacateiro, mangueira e goiabeira (Oliveira et al., 2001).

Estes insetos apresentam grande capacidade de dispersão e adaptação às diversas condições climáticas. Em regiões tropicais, a ocorrência de inúmeras gerações anuais e um grande número de indivíduos dificultam o controle desta praga, o que gera grande preocupação em relação aos impactos negativos que as medidas de controle adotadas podem ter sobre os recursos naturais e as barreiras fitossanitárias impostas por países importadores de frutas.

No Brasil, A. woglumi foi constatada inicialmente na área urbana de Belém, em 2001 (Oliveira et al., 2001). Até o início de 2008, estava restrita aos Estados do Amapá, Amazonas, Tocantins e Maranhão (Lemos et al., 2007), sendo constatada recentemente em São Paulo, nos municípios de Arthur Nogueira, Cosmópolis, Engenheiro Coelho, Holambra Limeira, Conchal, Paulínia e Mogi-Mirim (Raga \& Costa, 2008; Yamamoto et al., 2008) e ao norte de Goiás, em Porangatu e Campinorte (Info Agricultura, 2008).

Aleurocanthus woglumi foi registrada no Maranhão em setembro de 2003, em Boa Vista do Gurupi, Imperatriz e Bacabal, em pomares de citros, e em março de 2004, em Barra do Corda e São Luís, em citros e mangueiras (Lemos et al., 2006 ).

No Maranhão, a mosca-negra encontra-se distribuída em 53 municípios, causando danos em pomares cítricos, principalmente nos pomares de fundo de quintal, que se constituem em fonte de subsistência e, às vezes, de renda para os agricultores. Os prejuízos causados são elevados, e a maioria dos agricultores de baixa renda tem abandonado seus pomares, pois, em altas populações, $A$. woglumi debilita as plantas pela sucção contínua de seiva, levando-as ao murchamento e, muitas vezes, à morte (Lemos et al., 2007). Além disso, eliminam uma excreção açucarada, induzindo o aparecimento de fungos saprófitas (fumagina) que revestem folhas, frutos e ramos, reduzindo a fotossíntese, impedindo a respiração da planta e diminuindo o nível de nitrogênio nas folhas (Prates, 2002).

Por tratar-se de uma praga quarentenária A2 para o Brasil, segundo a Instrução Normativa do MAPA $n^{\circ} 41$, de julho de 2008, os danos causados por este inseto não se restringe apenas aos danos diretos provocados nos seus hospedeiros, mas também efeitos cumulativos, como fechamento do comércio exterior, aumento de custos para execução de medidas quarentenárias (barreiras e vigilância) e desenvolvimento de pesquisas e controle. Apesar dos riscos citados, poucas são as pesquisas sobre esse aleirodídeo. Dessa forma, este trabalho teve como objetivos avaliar a dinâmica populacional de $A$. woglumi ao longo de um ano para se definir a época de maior ocorrência e seus picos populacionais, bem como avaliar a distribuição dessa praga em plantas de Citrus spp., de acordo com os pontos cardeais primários (norte, leste, sul e oeste), em duas áreas de produção no município de São Luís - MA.

\section{MATERIAL E MÉTODOS}

O trabalho foi realizado sob condição de campo, em duas áreas no município de São Luís - MA. Uma área de produção não comercial, localizada na Fazenda-Escola do Centro de Ciências Agrárias que possui um total de 25 ha, sendo que o pomar de citros, com sete anos de idade, ocupa 0,5 ha onde são cultivadas as espécies Lima-ácida Tahiti (Citrus latifolia Tan.), Laranja-Natal-Folha-Murcha ( $C$. sinensis L. Obs.), Tangerina-Tanjaroa (C. reticulata Blanco), Limão-Rugoso-do-Maranhão (Citrus sp). e Limão-Galego (C. aurantifolia Swing.), todas enxertadas em Limoeiro-Cravo (Citrus limonia Osb.) e plantadas no espaçamento de $6 \times 6 \mathrm{~m}$, e coordenadas geográficas de $02^{\circ} 35^{\prime}$ 04,0” S e $44^{\circ} 12^{\prime}$ '33,3” W. O Limão-Rugoso-do-Maranhão e a tangerina-Tanjaroa são seleções locais coletadas no Estado. A área de produção comercial localiza-se no Polo de Itapera, ocupando 2 ha,onde são cultivadas as variedades Limão-Galego e Lima-ácida, com quatro anos de idade, ambas enxertadas em Limoeiro-Cravo em espaçamento de $6 \times 6 \mathrm{~m}$ e coordenadas geográficas $02^{\circ} 40^{\prime} 40,9$ ' S e 44 16' 51,4” W.

Durante todo o período de estiagem (agosto a dezembro), as plantas foram irrigadas pelo método de microaspersão e receberam os tratos culturais recomendados para a cultura, inclusive aplicação do ingrediente ativo imidacloprido, nos meses de julho e setembro de 2006 na área não comercial e metamidofós em junho de 2006 e parationa metílica em 
setembro de 2006 na área de produção comercial.

Os solos predominantes nessa região são os

Argissolos Vermelho-Amarelos, de baixa fertilidade natural e oriundos da formação Itapecuru (Moura, 1995), e o clima predominante na região é o úmido (classificação de Köeppen).

Para o estudo da dinâmica populacional da mosca-negra, selecionaram-se 10 plantas ao acaso em cada área, fazendo-se caminhamento em zigue-zague, sendo que cada ponto de amostragem foi constituído por uma planta. No período de julho de 2006 a junho de 2007, foram realizadas coletas quinzenais, sendo amostradas de cada planta 20 folhas da porção apical dos ramos, sendo cinco folhas de cada quadrante (norte, leste, sul e oeste), para a contagem do número de posturas, ovos e ninfas de $A$. woglumi, totalizando-se 200 folhas em cada área. As folhas coletadas foram colocadas em sacos plásticos devidamente identificados com data, local de coleta e quadrante. O material foi levado ao Laboratório de Entomologia da UEMA, onde as folhas foram conservadas em geladeira até a realização das contagens, com auxílio de um estereomicroscópio.

Para a análise da flutuação populacional, utilizou-se dos valores médios de cada variável (número de posturas, ovos e ninfas) para cada coleta, e para embasar o entendimento das curvas de flutuação obtidas, utilizou-se do somatório da precipitação pluvial em períodos quinzenais entre duas coletas. Com relação à distribuição de $A$. woglumi na planta, o delineamento utilizado foi o inteiramente casualizado, adotando-se o esquema fatorial 2 x 4 (período seco e chuvoso x quadrantes), com 13 repetições ( ${ }^{\circ}$ de coletas). Realizou-se uma análise exploratória de dados (procedimento Univariate, SAS ${ }^{\circledR}, 2001$ ), e as médias obtidas foram submetidas ao teste de Tukey, a 5\% de probabilidade (procedimento GLM, $\left.\mathrm{SAS}^{\circledR}, 2001\right)$.

\section{RESULTADOS E DISCUSSÃO}

Na área de produção não comercial, observou-se uma grande influência da precipitação pluvial sobre a densidade populacional da mosca-negra. No início das coletas (período seco, baixa precipitação pluvial), registrou-se elevado número de posturas, ovos e ninfas/planta quando comparada ao período chuvoso (Figura 1). O primeiro pico do número de posturas, ovos e ninfas/planta foi registrado na segunda quinzena de julho de 2006 (184,3 posturas/planta, 8.052,4 ovos/planta e 737,1 ninfas/planta), e o segundo pico, na segunda quinzena de setembro de 2006 (152,7 posturas/planta, 5.037,4 ovos/planta e 511,6 ninfas/planta). Após o segundo pico, houve consi- derável queda no número de posturas, ovos e ninfas/ planta. Devido às chuvas, suas densidades populacionais mantiveram-se baixas até o final das coletas, concordando com Figueredo (2002), ao relatar que, na Venezuela, os maiores níveis populacionais da mosca-negra-dos-citros ocorreram também durante a época seca. Resultado similar foi encontrado por Silva (2006), que ao avaliar a dinâmica populacional de Bemisia tabaci (Hemiptera: Aleyrodidae), em cultivos olerícolas, relatou que a precipitação foi o principal fator meteorológico que atuou sobre a densidade populacional dos estádios ninfais e dos adultos. No entanto, Leite et al. (2002) verificaram que as temperaturas mais elevadas favoreceram as populações de mosca-branca, não se detectando o efeito da chuva sobre este aleirodídeo.

Para as variáveis avaliadas número de posturas, ovos e ninfas/planta, houve diferença estatística dos quadrantes norte e sul, quando comparados aos quadrantes leste e oeste no período seco, verificando-se nesses quadrantes os maiores índices populacionais de $A$. woglumi (Tabela 1). Entretanto, no período chuvoso, para as mesmas variáveis, não foi observada preferência por quadrante, pois a praga distribuiu-se igualmente na copa da árvore, exceto em relação ao número de ninfas, onde houve diferença significativa do quadrante oeste em relação aos quadrantes sul e leste (Tabela 1). Cherry e Fitzpatrick (1979) e Dowell e Cherry (1981) observaram que, em relação à distribuição do inseto entre os quadrantes, não houve diferença estatística quando amostrados insetos vivos; entretanto, quando amostrados insetos mortos, o número de folhas infestadas foi estatisticamente diferente entre os quadrantes avaliados.

Na área de cultivo comercial, registrou-se um pico populacional na primeira quinzena de julho de 2006 (12,2 posturas/planta, 483,4 ovos/planta e 183,4 ninfas/planta) (Figura 2). Já a partir da segunda quinzena de julho, registrou-se decréscimo do número de posturas, ovos e ninfas/planta, mantendose baixos até a primeira quinzena de novembro de 2006. Na segunda quinzena de novembro de 2006 , foi constatado mais um pico do número de posturas e ovos (13,2 posturas/planta e 441,5 ovos/planta), exceto em relação ao número de ninfas. No período chuvoso, houve decréscimo no número de posturas, ovos e ninfas de A. woglumi. Situação inversa foi verificada por Yamamoto et al. (2001), estudando a flutuação populacional de Diaphorina citri (Hemiptera: Psyllidae) em pomares de citros do Estado de São Paulo, concluindo que a densidade mais alta do inseto no campo foi no período com maiores precipitações (dezembro e janeiro), e a mais baixa durante o período seco (junho, julho e agosto). Já 
Dengel (1981) afirmou que, no início da estação chuvosa, a população de mosca-branca é pequena, mas pode aumentar bruscamente, pois durante o período chuvoso haveria uma fuga do inseto para a parte abaxial da folha; entretanto, como as folhas jovens são preferíveis como alimento, os insetos poderiam voltar nos dias menos chuvosos.

Os quadrantes norte e sul diferiram estatisticamente dos quadrantes leste e oeste em relação ao número de posturas e ovos/planta no período seco (Tabela 2). Contrariamente, em relação ao número de ninfas, o inseto distribuiu-se na copa da planta de modo semelhante, não ocorrendo diferença significativa entre os quadrantes. No período chuvoso, não houve diferença na distribuição do número de posturas, ovos e ninfas nos quadrantes das árvores (Tabela 2).

Nas duas áreas, verificou-se que, no período chuvoso, houve diminuição da população de mosca-negra. Na área de produção não comercial, houve diferença significativa entre os dois períodos avaliados, observando-se que o número de posturas, ovos e ninfas foi maior no período seco, em todos os quadrantes. (Tabela 1). Por outro lado, na área de produção comercial, não houve diferença entre o período seco e o chuvoso, exceto no quadrante oeste para o número de posturas, ovos e ninfas, e no quadrante leste, para o número de ovos (Tabela 2). Lemos et al. (2003) também constaram que a infestação da mosca-branca na época das águas foi reduzida quando comparada à época seca. Da mesma forma, Menezes et al. (2008) observaram que chuvas intensas podem funcionar como importante fator de mortalidade de adultos de mosca-negra na cultura de citros, o que provavelmente afetaria o desenvolvimento das fases de ovo e ninfa.

Nas condições em que este trabalho foi realizado, a mosca-negra ocorreu em todos os meses do ano e com seus picos populacionais concentrando-se no período de baixa precipitação pluviométrica. Esses resultados favorecem o planejamento e a execução de medidas operacionais que sejam mais viáveis e seguras para o controle desse aleirodídeo dentro dos princípios do manejo integrado de pragas.

TABELA 1- Médias de posturas/planta, de ovos/planta e de ninfas/planta, nos períodos seco (PS) e chuvoso (PC), de Aleurocanthus woglumi Ashby (Hemiptera: Aleyrodidae) em Citrus spp., em área de produção não comercial. São Luís - MA, 2006-2007.

\begin{tabular}{|c|c|c|c|c|c|c|}
\hline \multirow[b]{2}{*}{ Quadrantes } & \multicolumn{2}{|c|}{$\mathrm{N}^{0}$ de posturas/planta } & \multicolumn{2}{|c|}{$\mathrm{N}^{o}$ de ovos/planta } & \multicolumn{2}{|c|}{$\mathrm{N}^{\mathrm{o}}$ de ninfas/planta } \\
\hline & PS & PC & PS & $\mathrm{PC}$ & PS & PC \\
\hline Norte & $132,54 \mathrm{bA}$ & $8,92 \mathrm{aB}$ & $4250,54 \mathrm{bA}$ & $211,08 \mathrm{aB}$ & 367,46 bA & $23,00 \mathrm{abB}$ \\
\hline Sul & $118,00 \mathrm{bA}$ & $8,15 \mathrm{aB}$ & $3802,08 \mathrm{bA}$ & $246,08 \mathrm{aB}$ & $301,38 \mathrm{bA}$ & 23,31 bB \\
\hline Leste & 208,23 aA & $9,54 \mathrm{aB}$ & 7156,08 aA & $253,15 \mathrm{aB}$ & 568,46 aA & 22,23 bB \\
\hline Oeste & 289,62 aA & $15,77 \mathrm{aB}$ & 9889,85 aA & $488,54 \mathrm{aB}$ & 812,31 aA & $46,31 \mathrm{aB}$ \\
\hline CV (\%) & & & & & & \\
\hline
\end{tabular}

Letras minúsculas na mesma coluna e maiúsculas na linha não diferem entre si, estatisticamente, pelo teste de Tukey, a 5\% de probabilidade.

TABELA 2- Médias de posturas/planta, de ovos/planta e de ninfas/planta, nos períodos seco (PS) e chuvoso (PC), de Aleurocanthus woglumi Ashby (Hemiptera: Aleyrodidae) em Citrus spp., em área de produção comercial. São Luís - MA, 2006-2007.

\begin{tabular}{crrrrrr}
\hline & \multicolumn{2}{c}{$\mathrm{N}^{\circ}$ de posturas/planta } & \multicolumn{2}{c}{$\mathrm{N}^{\circ}$ de ovos/planta } & \multicolumn{2}{c}{$\mathrm{N}^{\circ}$ de ninfas/planta } \\
\cline { 2 - 7 } Quadrantes & \multicolumn{1}{c}{ PS } & PC & PS & \multicolumn{1}{c}{ PC } & PS & PC \\
\hline Norte & $6,85 \mathrm{bA}$ & $5,85 \mathrm{aA}$ & $199,00 \mathrm{bA}$ & $182,77 \mathrm{aA}$ & $68,85 \mathrm{aA}$ & $40,54 \mathrm{aA}$ \\
Sul & $7,92 \mathrm{bA}$ & $3,46 \mathrm{aA}$ & $263,00 \mathrm{bA}$ & $95,23 \mathrm{aA}$ & $93,15 \mathrm{aA}$ & $43,38 \mathrm{aA}$ \\
Leste & $17,54 \mathrm{aA}$ & $3,77 \mathrm{aA}$ & $533,77 \mathrm{aA}$ & $97,38 \mathrm{aB}$ & $61,77 \mathrm{aA}$ & $30,85 \mathrm{aA}$ \\
Oeste & $16,23 \mathrm{aA}$ & $5,00 \mathrm{aB}$ & $511,46 \mathrm{aA}$ & $127,15 \mathrm{aB}$ & $80,92 \mathrm{aA}$ & $28,62 \mathrm{aB}$ \\
\hline CV $(\%)$ & \multicolumn{2}{c}{45,19} & \multicolumn{2}{c}{62,06} & \multicolumn{2}{c}{66,53} \\
\hline
\end{tabular}

Letras minúsculas na mesma coluna e maiúsculas na linha não diferem entre si, estatisticamente, pelo teste de Tukey, a 5\% de probabilidade. 


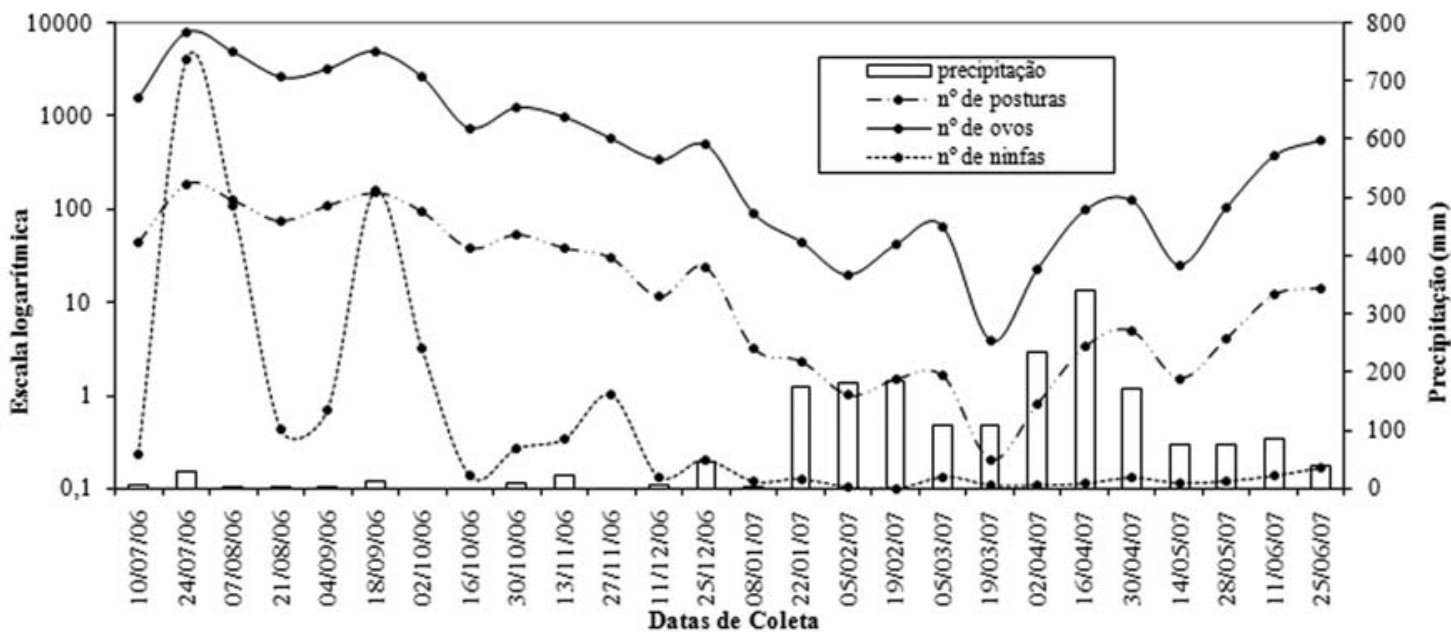

FIGURA 1 - Relação da precipitação pluvial e do número médio de posturas/planta, de ovos/planta e de ninfas/planta de Aleurocanthus woglumi Ashby (Hemiptera: Aleyrodidae) em Citrus spp., em área de produção não comercial. São Luís - MA, 2006-2007.

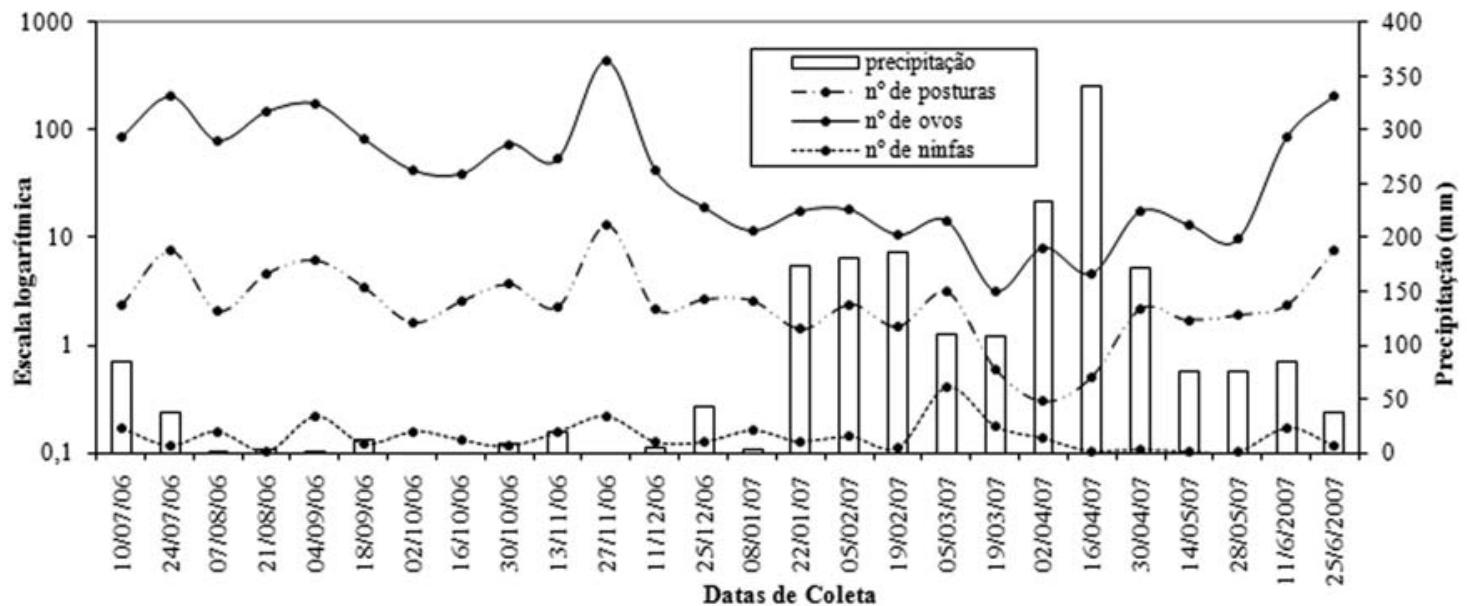

FIGURA 2- Relação da precipitação pluvial e do número médio de posturas/planta, de ovos/planta e de ninfas/planta de Aleurocanthus woglumi Ashby (Hemiptera: Aleyrodidae) em Citrus spp.,em área de produção comercial. São Luís - MA, 2006-2007.

\section{CONCLUSÕES}

1-Os maiores níveis populacionais de $A$. woglumi ocorreram no período de baixa precipitação pluviométrica.

2-A distribuição do número de posturas, ovos e ninfas de A. woglumi foi semelhante nos quadrantes norte, leste, sul e oeste das árvores no período chuvoso.

3-Houve maior concentração de posturas, ovos e ninfas de A. woglumi nos quadrantes leste e oeste no período seco.

\section{AGRADECIMENTOS}

À FAPEMA (Fundação de Amparo à Pesquisa e ao Desenvolvimento Tecnológico do Maranhão), pela concessão da bolsa.

\section{REFERÊNCIAS}

CHERRY, R.; FITZPATRICK, G. Intra-tree dispersion of citrus blackfly. Environmental Entomology, Maryland, v. 8, n. 6, p. 997 - 999, dez. 1979. 
DENGEL, H.J. Investigations on the incidence of Bemisia tabaci (Genn.) adults on different cassava varieties. Plant Research and Development, Tubingen v. 1, n. 14, p. 37 - 49, 1981.

DOWELL, R.V.; CHERRY, R.H. Detection of, and sampling procedures for, the citrus blackfly in urban Southern Florida. Researches on Population Ecology, Kyoto, v. 23, n. 1, p. 19 -26, 1981.

FIGUEREDO, L.C. Manejo fitossanitario de la mosca prieta de los citricos (Aleurocanthus woglumi Ashby) en las condiciones de la empresa de citricos sola. 2002. 63 f. Dissertação (Maestría en Fruticultura Tropical) - Instituto de Investigaciones de Fruticultura Tropical, La Havana, 2002.

INFO AGRICULTURA. SP solicita revisão de legislação federal para mosca-negra-dos-citros. Informativo Semanal, São Paulo, v.2, n.7, 2008. Disponível em: <http://www.agricultura.sp.gov.br/ INFORMATIVO/Info\%2025/alta.pdf > Acesso em: 20 abr. 2008.

LEITE, G.L.D.; PICANCO, M.; ZANUNCIO, J.C.; MOREIRA, M.D.; PEREIRA, P.R. Fatores que influenciam no ataque de mosca-branca em jiloeiro. Pesquisa Agropecuária Brasileira, Brasília, v. 37, n. 7, p. 1033 - 1037, 2002.

LEMOS, L.B.; FILHO, D.F.; SILVA, T.R.B.; SORATTO, R.P. Suscetibilidade de genótipos de feijão ao vírus-do-mosaico-dourado. Pesquisa Agropecuária Brasileira, Brasília, v. 38, n. 5, p. 575 - 581, 2003.

LEMOS, R.N.S.; SANTANA, G.F.; MEDEIROS, F.R. Mosca-Negra-dos-Citros Aleurocanthus woglumi (Hemiptera: Aleyrodidae) - Situação e Controle no Maranhão. In: SIMPÓSIO DE MANGA DO VALE DO SÃO FRANCISCO, 2., 2007, Juazeiro. Palestras... Petrolina: Embrapa SemiÁrido, 2007. CD-ROM

LEMOS, R.N.S.; SILVA, G.S.; ARAÚJO, J.R.G.; CHAGAS, E.F.; MOREIRA, A.A.; SOARES, A.T.M. Ocorrência de Aleurocanthus woglumi Ashby (Hemiptera: Aleyrodidae) no Maranhão. Neotropical Entomology, Londrina, v. 35, n. 4, p. 558 - 559, 2006.

MENEZES, R.O.; MAIA, P.S.P.; FARIAS, P.R.S.; MONTEIRO, B.S.; ALBUQUERQUE, G.D.P. Amostragem sequencial com base na lei de Taylor para levantamento populacional da mosca-negra-doscitros (Aleurocanthus woglumi Ashby) em pomares de citros no município de Capitão Poço-PA. In: SEMINÁRIO DE INICIAÇÃO CIENTÍFICA DA UFRA, 6., SEMINÁRIO DE INICIAÇÃO CIENTÍFICA DA EMBRAPA AMAZÔNIA ORIENTAL, 6., 2008, Belém. Anais... Bélem: UFRA/EMPRAPA AMAZÔNIA ORIENTAL. 2008. Disponível em: $<$ http://anaispibic2008.cpatu.embrapa.br/index2. html>. Acesso em: 21 fev. 2008.

MOURA, E.G. Atributos físico-hídricos e fertilidade de um PVA Distrófico da formação Itapecuru em São Luís-MA, que afetam o crescimento do milho (Zea mays L.). 1995. 68 f. Tese (Doutorado em Agronomia - Irrigação e Drenagem) - Faculdade de Ciências Agronômicas, Universidade Estadual Paulista, Botucatu, 1995.

OLIVEIRA, M.R.V.de; SILVA, C.C.A. da; NÁVIA D. Mosca-negra-dos-citros Aleurocanthus woglumi: alerta quarentenário. Brasília: Ministério da Agricultura, Pecuária e Abastecimento, 2001. 12 p.

PRATES, H.S. Mosca-negra. 2002. CECOR/CATI. Disponível em: <http:/www.cati.sp.gov.br/novacati/ tecnologias/catiresponde/cr49_mosca_negra.htm>. Acesso em: 26 mar. 2008.

RAGA, A.; COSTA, V.A. Mosca-negra-dos-citros. São Paulo: Agência Paulista de Tecnologia dos Agronegócios, 2008. p. 1-9. (Documento Técnico, 001)

SAS INSTITUTE INC. SAS System for Microsoft Windows 98. Cary, 2001.

SILVA, M.C. Dinâmica populacional e variabilidade genética da mosca-branca Bemisia tabaci (Gennadius, 1889) biótipo B (Hemiptera: Aleyrodidae) em cultivos olerícolas em São Luís -MA. 2006. 91 F. Dissertação (Mestrado em Agroecologia) - Universidade Estadual do Maranhão, São Luís, 2006.

YAMAMOTO, P.T.; LOPES, S.; BASSANEZI, R.B.; BELASQUE JUNIOR, J.; SPOSITO, M.B. Citros: estrago à vista. Cultivar Hortaliças e Frutas, Pelotas, v.8, p.22 - 24, 2008.

YAMAMOTO, P.T.; PAIVA, P.E.B.; GRAVENA, S. Flutuação populacional de Diaphorina citri Kuwayama (Hemiptera: Psyllidae) em pomares de citros na região norte do Estado de São Paulo. Neotropical Entomology, Londrina, v. 30, n.1, p. 165 - 170, 2001. 